Article

\title{
Improving Spectral Efficiency in the SCMA Uplink Channel
}

\author{
Sergio Vidal-Beltrán * (1) and José Luis López-Bonilla \\ Instituto Politécnico Nacional, Escuela Superior de Ingeniería Mecánica y Eléctrica-Zacatenco, \\ 07738 Ciudad de México, Mexico; jlopezbo@ipn.mx \\ * Correspondence: svidalb@ipn.mx; Tel.: +52-5557296000
}

check for updates

Citation: Vidal-Beltrán, S.;

López-Bonilla, J.L. Improving Spectral Efficiency in the SCMA Uplink Channel. Mathematics 2021, 9, 651. https://doi.org/10.3390/ math9060651

Academic Editors: Rashid Ali and Manuel Alberto M. Ferreira

Received: 11 February 2021

Accepted: 16 March 2021

Published: 18 March 2021

Publisher's Note: MDPI stays neutral with regard to jurisdictional claims in published maps and institutional affiliations.

\begin{abstract}
The Third Generation Partnership Project (3GPP) and the International Telecommunication Union (ITU) identified the technical requirements that the fifth generation of mobile communications networks (5G) had to meet; within these parameters are the following: an improved data rate and a greater number of users connected simultaneously. 5G uses non-orthogonal multiple access (NOMA) to increase the number of simultaneously connected users, and by encoding data it is possible to increase the spectral efficiency (SE). In this work, eight codewords are used to transmit three bits simultaneously using Sparse Code Multiple Access (SCMA), and through singular value decomposition (SVD) the Euclidean distance between constellation points is optimized. On the other hand, applications of machine intelligence and machine intelligence in 5G and beyond communication systems are still developing; in this sense, in this work we propose to use machine learning for detecting and decoding the SCMA codewords using neural networks. This paper focuses on the Use Case of enhanced mobile broadband (eMBB), where higher data rates are required, with a large number of users connected and low mobility. The simulation results show that it is possible to transmit three bits simultaneously with a low bit error rate (BER) using SVD-SCMA in the uplink channel. Our simulation results were compared against recent methods that use spatial modulation (SM) and antenna arrays in order to increase spectral efficiency. In adverse Signal-to-Noise Ratio (SNR), our proposal performs better than SM, and antenna arrays are not needed for transmission or reception.
\end{abstract}

Keywords: 5G; enhanced mobile broadband (eMBB); neural networks; non-orthogonal multiple access (NOMA); supervised learning; sparse code multiple access (SCMA); singular value decomposition (SVD); uplink channel biointerfaces

\section{Introduction}

The requirements and the specific needs that should be met by a fifth generation (5G) mobile communication system were initially collected by the Third Generation Partnership Project (3GPP) in [1], and more than seventy Use Cases were identified and compiled them into five main categories, which are: Enhanced Mobile Broadband (eMBB), Massive Machine Communications/mIoT Massive Internet of Things (mMTC/mIoT), Critical Communications/Ultra Reliable Low Latency Communications (CriC/URLLC), Enhanced Vehicle-to-Everything (eV2X), and the final category is Network Operation NEO. The eMBB category is oriented to give access to content, services and multimedia data to the human being. This category includes, in the first instance, hotspots, characterized by having high user density, high capacity and low user mobility. In the second instance, it gives broad coverage to users with medium to high speeds and an improved transmission data rate. This work is oriented to eMBB where a high user density, low mobility and high transmission rates are required, that as a result is an improved spectral efficiency.

In conventional Orthogonal Multiple Access (OMA) schemes, used in 4G, users occupy radio resources that are orthogonal in time, frequency or code. Ideally, there is no interference between multiple users due to orthogonal resource allocation in OMA, so 
simple single-user detection can be used to separate signals from different users. Theoretically, it is known that OMA does not achieve the maximum joint transmission rate in multiuser wireless systems [2]; in addition, in conventional OMA schemes, the maximum number of users is limited by the total number and the granularity of orthogonal resources. For fifth generation communication systems (5G) and beyond, Non-Orthogonal Multiple Access (NOMA) is used, which improves the performance of OMA systems. NOMA allows controllable interference by allocating non-orthogonal resources with increasing receiver complexity. Compared to OMA, the main advantages of NOMA include improving spectral efficiency and connecting a greater number of users, among others.

There are different NOMA access techniques, among which is Sparse Code Multiple Access (SCMA) [3,4], which directly encodes user data into multidimensional codewords using low-density codebooks. With this method, each user is assigned to a different codebook and the data within is used to map the user's bits directly to a low-density vector (sparse vector) called a codeword [5-7]. Each vector contains the zero value in the same two dimensions of all its elements, and the position of the zeros is determined randomly to prevent collisions between users [8-10]. There are different methods to design SCMA codebooks [5-10]; in [11], we proposed using Singular Value decomposition (SVD) to improve signal detection and decoding even under adverse electromagnetic propagation conditions.

Originally, SCMA transmits two bits on the uplink using one transmitting antenna and one receiving antenna. Once with SVD-SCMA the distances between the codewords are optimized, the next challenge is to increase the spectral efficiency by transmitting more bits simultaneously. Recently, spatial modulation $[12,13]$ has been used to improve the SE in NOMA systems [14-16]. SM increases the SE by allocating part of the input data stream (spatial symbol), to activate an antenna to transmit the modulation symbol. Another approach is to increase the number of bits that are encoded per codewords, which makes it more difficult to detect and decode signals at the receiver. Considering the traditional SCMA approach of six users, four radio carriers, and two bits encoded in four codewords, where each carrier carries data from three users and each user sends its information on two carriers, each pair of bits can be represented by up to 16 different combinations. If the number of bits to encode is increased to three, then each bit triplet could be represented by up to 64 combinations, producing a total IQ constellation of 512 different points to transmit 3 bits simultaneously.

On the receiver side [17-20], to detect and decode the SCMA codewords, two techniques are mainly used, on the one hand Message Passing Algorithm (MPA) and maximum likelihood (ML), and on the other hand the minimum Euclidean distance method is used. In [11], the authors proposed using a neural network-based receiver, using supervised learning for the detection and decoding of SCMA signals.

Finally, we can mention that the authors in $[14,15]$ are using multiple antennas and spatial modulation to increase spectral efficiency. In [11] we showed that SVD creates codebooks with better performance than its predecessors; the motivation for this work is to demonstrate that it is possible to increase spectral efficiency by maintaining the configuration of one transmitting antenna and one receiving antenna, taking up and expanding the application of SVD to the design and construction of NOMA codebooks for communications systems beyond 5G.

\section{Related Work and Contributions}

This work is part of an ongoing project. In [11] it was proposed to use SVD for the design and construction of SCMA codebooks; later it was proposed in [21,22] to use neural networks in the receiver to improve performance in the detection and decoding of SCMA codewords, even under adverse SNR conditions. In this work, the scope of SVD-SCMA is expanded, showing that it is possible to increase spectral efficiency. The performance of the method proposed in this work was compared with recent proposals that use spatial modulation and antenna arrays to increase spectral efficiency. 
SVD-SCMA produces codebooks with an optimal distance between the points of the codeword, which improves bit error rate (BER). In the works described in [5-11], four codewords $(M=4)$, are encoded into two bits. The authors in [14-16] proposed to use SM-SCMA to increase SE. In SM-SCMA, the information of each user is divided in two parts; one part is used to select the active transmitting antenna and the other part is directly assigned to an SCMA codeword according to each user's codebook. Detection and decoding at the receiver estimates both spatial and modulated symbols by performing an exhaustive search or by using one of the low-complexity decoding algorithms. These systems can transmit three bits at a time: a 2-bit spatial symbol and 1-bit modulated symbol, using more than one antenna. In [16], the authors proposed Rotational Generalized Spatial Modulation (RGSM-SCMA), achieving an SE and BER similar to SM-SCMA using a lower number of antennas by activating more than one antenna at a time. In this work, we propose three-bit encoding using a combination of Singular Value Decomposition SCMA (SVD-SCMA) [11] with an 8-APSK constellation. The SVD-SCMA performance is compared to the phase rotation method [5-7], SM-SCMA and RGSM-SCMA [16]. For the detection and decoding of SM-SCMA and RGSM-SCMA, MPA was used while for SVD-SCMA a receptor based on neural networks was implemented. The results of this work show that it is possible to increase the spectral efficiency of the uplink with a lower BER using only one transmitting antenna and one receiving antenna.

This work is organized as follows: In the next section the method to design the SVDSCMA codebooks is described; later, the receiver used in this work is addressed, which is based on neural networks, as well as the training stage of the neural network. Next, the results of this work are shown. First, the results of the training stage of the neural network are described and later on the BER performance compared with SM-SCMA and RGSM-SCMA. Finally, the conclusions are described, and the final considerations of this work are discussed, as well as possible extensions of it.

\section{System Model and Problem Formulation}

This work starts with the assumption that it is possible to use eight codewords $(M=8)$ to encode three bits using 8-APSK modulation [23] (Amplitude and phase-shift keying), which is defined by Equation (1):

$$
X=\left\{\begin{array}{c|c}
r_{1} e^{i\left(\varphi_{1}+\frac{2 \pi}{n_{1}} k\right)} \mid k=0,1, \ldots n_{1}-1 \\
r_{2} e^{i\left(\varphi_{2}+\frac{2 \pi}{n_{2}} k\right)} \mid k=0,1, \ldots n_{2}-1 \\
\vdots \\
r_{N} e^{i\left(\varphi_{N}+\frac{2 \pi}{n_{N}} k\right)} \mid k=0,1, \ldots n_{N}-1
\end{array}\right.
$$

where $N$ is the number of concentric rings $(N=2), n$ the number of points per ring $\left(n_{1}=n_{2}=4\right), r$ is the radius of the circle, $\left(r_{1}=1.41, r_{2}=4.24\right)$, and $\varphi$ is the offset angle $\left(\varphi_{1}=\varphi_{2}=\frac{\pi}{4}=45^{\circ}\right)$, which leads a constellation of eight symbols. Based on [16], to build the codebooks the following technical considerations and constrains are applied: there will always be more users than radio resources, four radio subcarriers (SC) are used $(K=4)$, six users $(J=6)$, two code dimensions $(N=2)$, three users sharing the same subcarrier $\left(d_{f}=3\right)$ and each user uses two subcarriers to transmit their information $\left(d_{v}=2\right)$, codebooks are an array of $4 \times 4$, where the elements of two rows are zeros. The factor graph (FG) defines which subcarriers are using each user, which in its matrix representation is given by Equation (2).

$$
\mathrm{F}=\left[\begin{array}{cccccc}
\varphi_{1} & 0 & \varphi_{2} & 0 & \varphi_{3} & 0 \\
\varphi_{2} & 0 & 0 & \varphi_{3} & 0 & \varphi_{1} \\
0 & \varphi_{2} & \varphi_{1} & 0 & 0 & \varphi_{3} \\
0 & \varphi_{3} & 0 & \varphi_{1} & \varphi_{2} & 0
\end{array}\right]
$$


where $\varphi$ is the rotation angle for different users, obtained from Equation (3):

$$
\varphi_{u}=(u-1) \frac{2 \pi}{M d_{f}}+e_{u} \frac{2 \pi}{M}, \forall u=1, \ldots d_{f}
$$

According to [16], the SCMA codebooks are obtained from Equation (4):

$$
X_{j}=V_{j} \Delta_{j} V_{S V D^{\prime}}^{*} \mid j=1,2, \ldots J
$$

$X_{j}$ are the codebooks for $J-$ users, $\Delta_{j}$ is a rotation operator, which is defined by Equation (5) and (6):

$$
\begin{array}{cc}
\Delta_{j}=\operatorname{diag}\left(f_{j}\right)=f_{j}, \mid j=1,2, \ldots, 6 \\
\Delta_{1}=\left[\begin{array}{cc}
\varphi_{1} & 0 \\
0 & \varphi_{2}
\end{array}\right] \quad \Delta_{2}=\left[\begin{array}{cc}
\varphi_{2} & 0 \\
0 & \varphi_{3}
\end{array}\right] \quad \Delta_{3}=\left[\begin{array}{cc}
\varphi_{2} & 0 \\
0 & \varphi_{1}
\end{array}\right] \\
\Delta_{4}=\left[\begin{array}{cc}
\varphi_{3} & 0 \\
0 & \varphi_{1}
\end{array}\right] \quad \Delta_{5}=\left[\begin{array}{cc}
\varphi_{3} & 0 \\
0 & \varphi_{2}
\end{array}\right] \quad \Delta_{6}=\left[\begin{array}{cc}
\varphi_{1} & 0 \\
0 & \varphi_{3}
\end{array}\right]
\end{array}
$$

$V_{j}$ is a dispersion matrix for each user which contains $K-N$ rows with zero value, is given by Equation (7):

$$
V_{1}=\left[\begin{array}{l}
10 \\
01 \\
00 \\
00
\end{array}\right] V_{2}=\left[\begin{array}{l}
00 \\
00 \\
10 \\
01
\end{array}\right] V_{3}=\left[\begin{array}{l}
10 \\
00 \\
01 \\
00
\end{array}\right] V_{4}=\left[\begin{array}{l}
00 \\
10 \\
00 \\
01
\end{array}\right] V_{5}=\left[\begin{array}{l}
10 \\
00 \\
00 \\
01
\end{array}\right] V_{6}=\left[\begin{array}{l}
00 \\
10 \\
01 \\
00
\end{array}\right]
$$

To calculate $V_{S V D}^{*}$, we start calculating the mother constellation $\left(M_{C}\right)$, the first vector $\left(S_{1}\right)$ of the mother constellation is given by Equation $(8)$ :

$$
\begin{aligned}
& S_{11}=1+1 i, S_{12}=-1+1 i, S_{13}=-1-1 i, S_{14}=1-1 i \\
& S_{15}=3+3 i, S_{16}=-3+3 i, S_{17}=-3-3 i, S_{18}=3-3 i
\end{aligned}
$$

The second dimension $S_{2}$ is the result of multiplying $U_{N} S_{1}$, where $U_{N}=\operatorname{diag}\left(1 e^{i \theta_{l-1}}\right)$ $\in C^{N \times M}$ and $\theta_{l-1}=\frac{(l-1) \pi}{M N}, l=1, \ldots, N$, so that $M_{C}$ is given by Equation (9):

$$
M_{C}=\left(s_{1}, S_{2}\right)^{T}=\left[\begin{array}{llll}
s_{11} & s_{12} & \ldots & s_{18} \\
s_{21} & s_{22} & \ldots & s_{28}
\end{array}\right]
$$

SVD is applied to the matrix defined in Equation (9), as shown in Equation (10):

$$
M_{S V D}=S V D\left(M_{C}\right)=U_{n \times p} \Sigma_{p \times p} V_{m \times p}^{*}
$$

$U$ and $V^{*}$ are orthonormal, which results in an optimal Euclidean distance between each point, that is fundamental to define the final constellation of SCMA. $V_{S V D}^{*}$; which is a matrix of $2 \times 8$ similar to $M_{\mathcal{C}}$, with the difference that $V_{S V D}^{*}$ is a set of orthonormal eigenvectors. $X_{j}$ is a matrix of $4 \times 8$ that represents the six codebooks $(J=6)$, where the rows represent the subcarriers and the columns the eight codewords; two rows are zero according to the scatter matrix $V_{j}$. The works shown in [4-11] encoded two user bits in SCMA codewords, and the distance between adjacent points in the mother constellation is fundamental for the detection and decoding of the codewords. In this work, unlike its predecessors, each SCMA codeword represents three bits, producing an IQ constellation with closest adjacent points; Figure 1 shows the spacing between points of the constellation using SVD and phase rotation methods. 


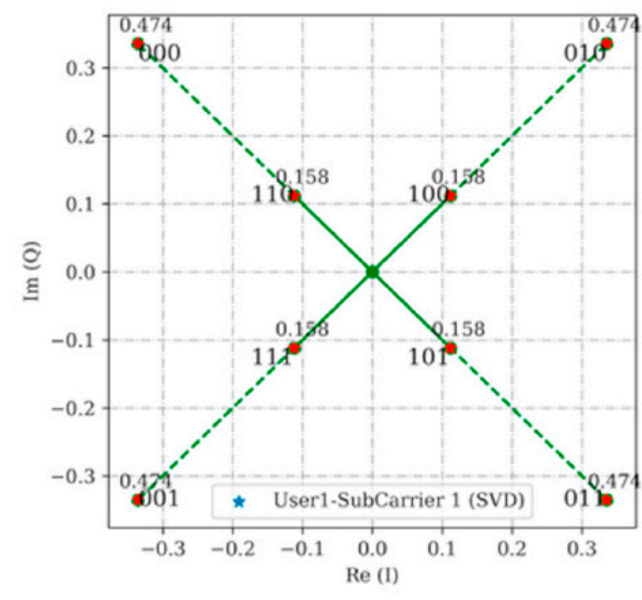

(a) Spacing between points of the constellation using SVD

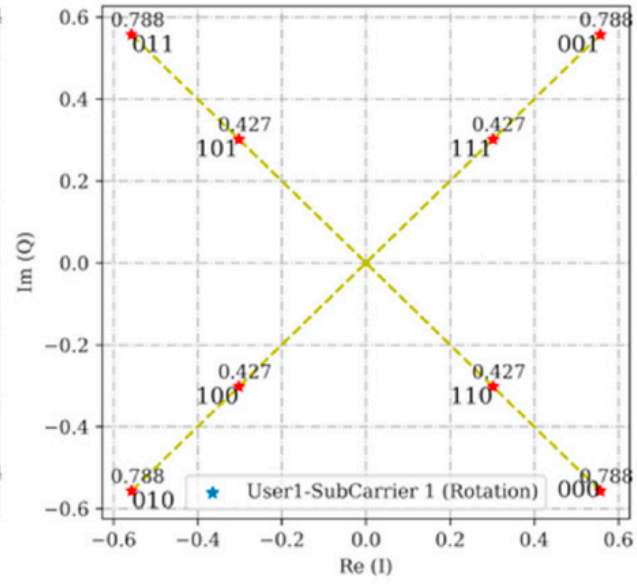

(b) Spacing between points of the constellation using Phase rotation

Figure 1. Minimum distance between points of the mother constellation.

According to Figure 1a, the distance ratio between adjacent points of the SVD constellation is $\frac{0.474}{0.158}=3$, while from Figure $1 \mathrm{~b}$ it is observed that the distance ratio in the constellation by phase rotation is $\frac{0.788}{0.427}=1.84$. When SVD is applied to the mother constellation, the resulting matrixes are orthogonal with orthonormal vectors, which produces constellations with improved distance between adjacent points. According to the FG showed in (2), the SC1 carries data of the Users 1, 3 and 5; each three-bit codeword could be represented by 64 possible combinations, since each codeword (three bits) of User 1 could be combined with the eight possible values of User 3 and the eight possible values of User 5, with the result that eight codewords for each user could be represented by up to 512 combinations. The IQ constellation is shown in Figure 2.

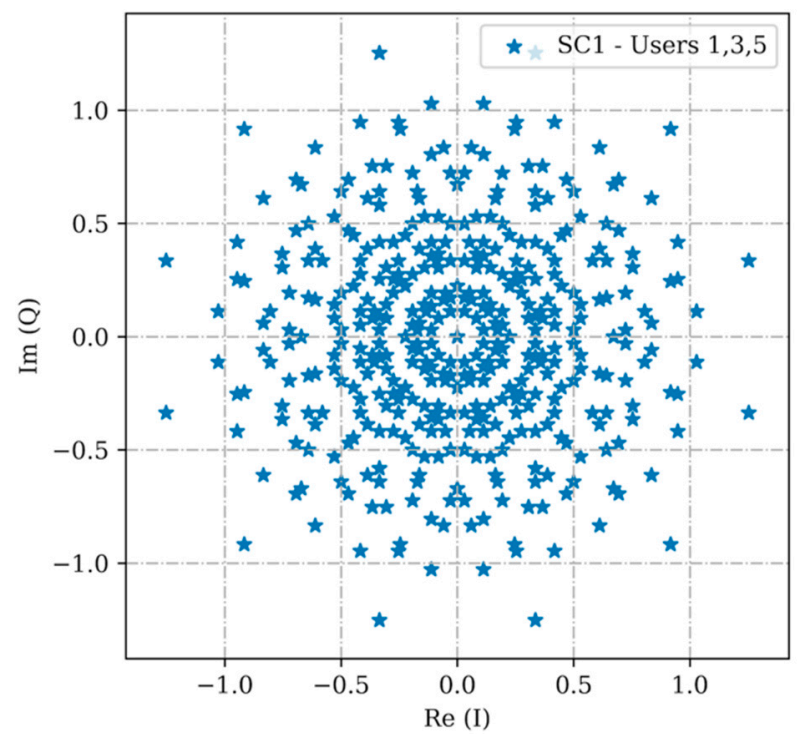

Figure 2. Subcarrier 1 IQ constellation.

\section{SCMA Receiver}

At the receiver, the detection and decoding of the SCMA codewords are directly dependent on the design of the codebooks and channel noise. In [4-10], MPA and minimum Euclidean distance are used for detection and decoding. In [11] and [21], supervised learning using neural networks is used for uplink detection and decoding with four codewords (two bits). In this work a neural network was used as shown in Figure 2. 
The resource allocation shown in Equation (2), defines which subcarrier each user uses to send their data. From Figure 3, it is observed that User 1 will use SC 1 and SC 2 (solid lines).

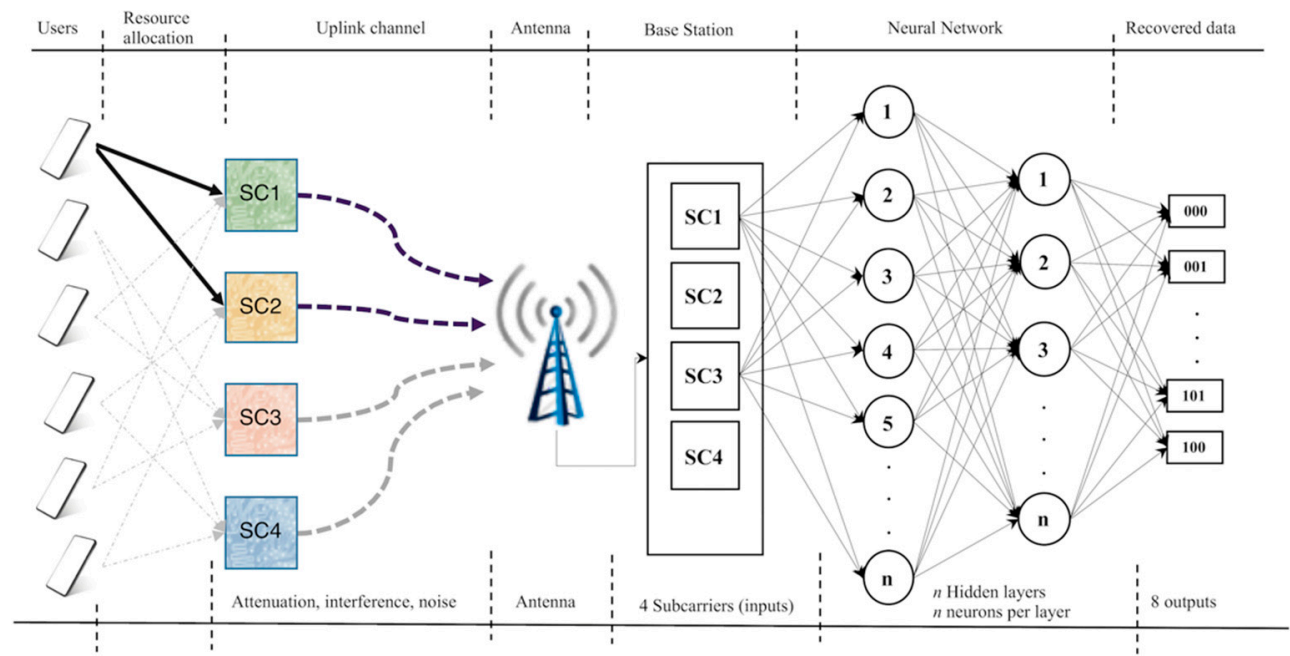

Figure 3. Neural network-based receptor diagram.

The codebook resulting from Equation (10) is given by Equation (11), where it is also observed that only the first two rows are occupied with the data of each codeword.

$$
\left[\begin{array}{c}
-0.34+0.34 j-0.11+0.11 j 0.11-0.11 j 0.34-0.34 j 0.34+0.34 j 0.11+0.11 j-0.11-0.11 j-0.34-0.34 j \\
-0.01-0.16 j 0.03+0.47 j-0.03-0.47 j 0.01+0.16 j-0.16+0.01 j 0.47-0.03 j-0.47+0.03 j 0.16-0.01 j \\
0+0 . j 0+0 . j 0+0 . j 0+0 . j 0+0 . j 0+0 . j 0+0 . j 0+0 . j \\
0+0 . j 0+0 . j 0+0 . j 0+0 . j 0+0 . j 0+0 . j 0+0 . j 0+0 . j
\end{array}\right]
$$

The data in each codebook represent the neural network inputs in the receiver. The configuration of the neural network used in this work is shown in Table 1.

Table 1. Neural network configuration parameters.

\begin{tabular}{cc}
\hline Inputs & $\mathbf{4}$ (Subcarriers) \\
\hline Outputs & $8(3 \mathrm{bits})$ \\
Dense hidden layers & $7(1024,1024,512,256,128,64$ and 32 neurons) \\
Optimization algorithm & Adaptive Moment Estimation (Adam) [24] \\
Batch size & 50 \\
Validation interval & 0.08 \\
$\beta_{1}$ & 0.9 \\
$\beta_{2}$ & 0.999 \\
learning rate $\left(l_{r}\right)$ & 0.002 \\
Activation function & Relu (except for the last layer where SOFTMAX was used) \\
\hline
\end{tabular}

It is assumed that the base station knows the codebooks of all users and the resource allocation given by Equation (2). The precision of the neural network depends on the training phase. The training data set is dependent on number of codewords $(M)$, users per subcarrier $\left(d_{f}\right)$ and SNR range, as shown in Equation (12).

$$
\text { dataset }=M^{d_{f}} \times S N R(\text { range })
$$

Configuration parameters of the training stage are shown in Table 2. 
Table 2. Configuration parameters of the training stage.

\begin{tabular}{cc}
\hline Codewords (M) & $\mathbf{8}$ \\
\hline Users per subcarrier $\left(d_{f}\right)$ & 3 \\
SNR range & From $-20 \mathrm{~dB}$ up to $+20 \mathrm{~dB}$ \\
Channel & Rayleigh fading channel \\
Total training data set & 100 \\
Iterations & 50 times the data set shown in Equation (12) \\
\hline
\end{tabular}

Figure 4 shows the neural network learning precision for the SVD and phase rotation methods. The neural network with SVD-SCMA reached an accuracy of $93.96 \%$, while SCMA by phase rotation had $94.71 \%$.

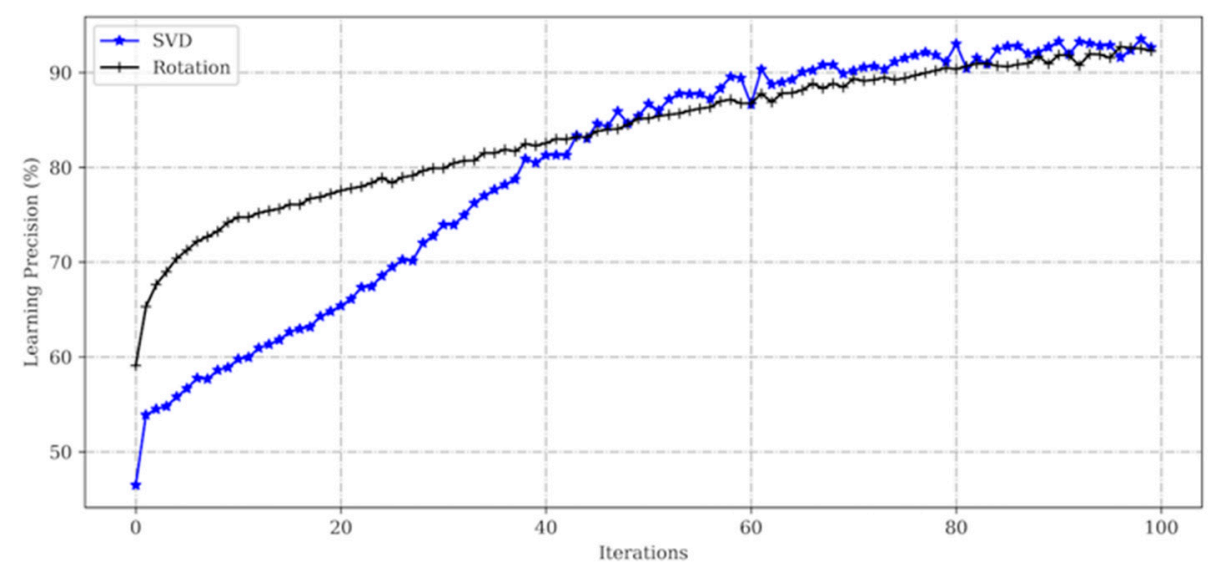

Figure 4. Neural network learning precision.

\section{Computational Complexity Analysis}

In general terms, the computational complexity for detection and decoding in the receiver depends on the number of transmitted bits $(b)$ and the number of users per subcarrier $\left(d_{f}\right)$; the complexity of both SM-SCMA and RGSM-SCMA [16] is given by $\mathcal{O}\left(2^{b d_{f}}\right)$. If multiple antennas $\left(N^{T}\right)$, are used, the complexity increases to $\left(N^{T}\right)^{2} \mathcal{O}\left(2^{b d_{f}}\right)$. On the other hand, the computational complexity of neural networks [25-27] that use backpropagation is defined in general terms as $\mathcal{O}\left(n^{5}\right)$ assuming that we have square matrices and that we have the same number of neurons for each hidden layer of the neural network, so the above expression could be expanded to $\mathcal{O}\left(n^{3} \times W_{j i} \times\right.$ epochs $)$, where $W_{j i}$ is the matrix of the activation function from layer $i$ to the layer $j$ and epochs is a hyperparameter of gradient descent that controls the number of complete passes through the training dataset. One way of making algorithms run faster is by using parallel execution, for example, running the matrix operations using Graphics Processing Unit (GPU).

GPUs are specifically designed to run many matrix operations in parallel since 3D geometry and animation can be expressed as a series of linear transformations. This is also why we usually train neural networks on GPUs. Since neural network resides in the Base station, it would be easier to deploy GPU in the receiver circuit.

\section{Simulation Results and Discussion}

Once the neural network was trained, the receiver performance was verified using ten million of new random bit triplets with different SNRs (from $-20 \mathrm{~dB}$ to $+20 \mathrm{~dB}$ ), and a Rayleigh fading channel was considered between the transmitter and the receiver. Figure 5 shows the precision in the predictions for SVD-SCMA and phase rotation. 


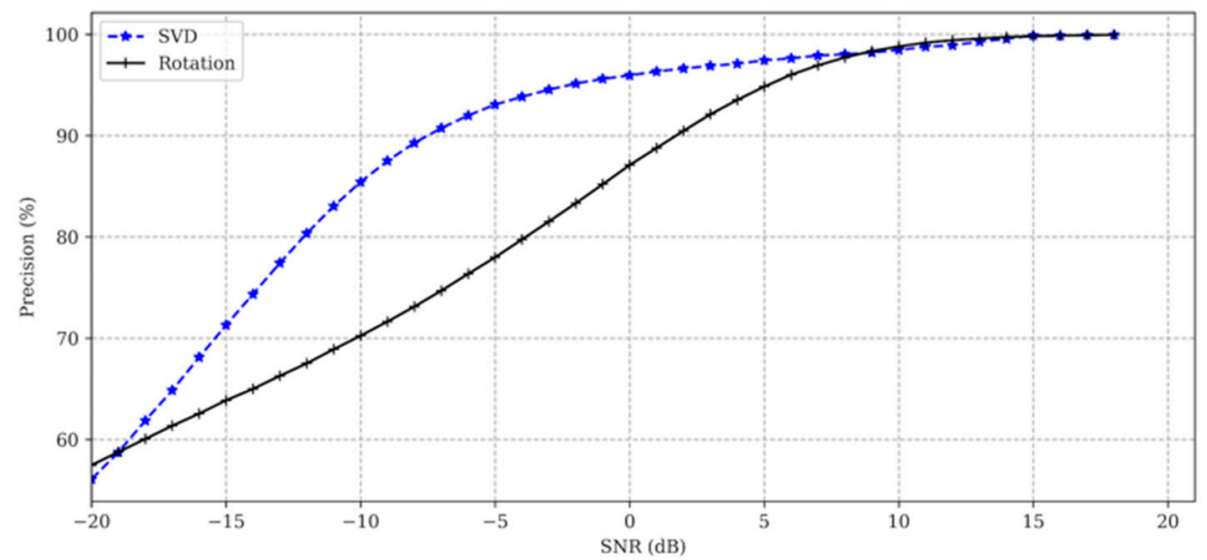

Figure 5. Precision of predictions.

To achieve $98 \%$ accuracy, SVD-SCMA requires an SNR of $+5 \mathrm{~dB}$, while phase rotation requires an SNR of $+8 \mathrm{~dB}$. Figure 4 shows that SVD-SCMA performs better under adverse SNR conditions (between -15 and $+8 \mathrm{~dB}$ ).

Figure 6 shows the BER performance comparison for SVD-SCMA and phase rotation; additionally, the SVD-SCMA performance using MPA (red line) is shown. It can be seen, from Figure 6, that SVD-SCMA has a better performance under adverse noise conditions (between $-15 \mathrm{~dB}$ and $+8 \mathrm{~dB}$ SNR); to achieve $2 \%$ errors, SVD-SCMA needs a SNR equal to $+5 \mathrm{~dB}$ while phase rotation requires $+8 \mathrm{~dB}$. For SNRs close to $+20 \mathrm{~dB}$, both methods have a similar performance. On the other hand, it is appreciated that MPA begins to decode from an SNR of $+3 \mathrm{~dB}$; this is due to the high complexity of the IQ constellation since it could exist up to 512 different values.

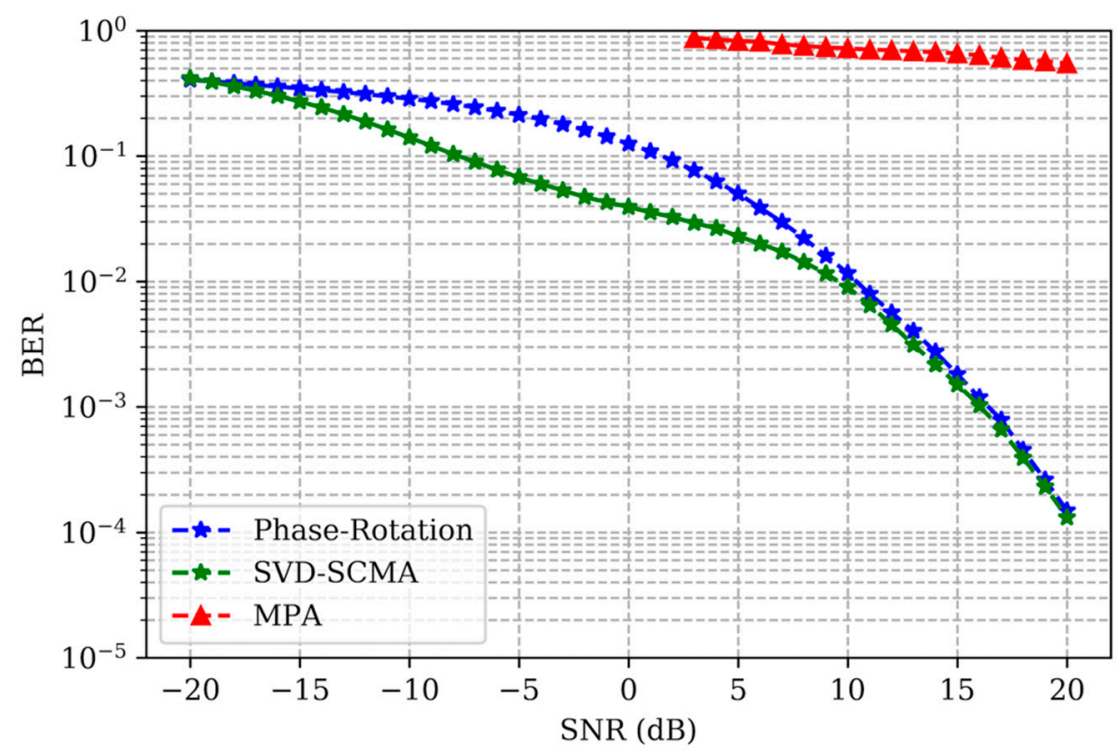

Figure 6. Bit error rate (BER) performance comparison.

SVD-SCMA and phase rotation methods rely on the design of codebooks using only one transmitting antenna and one receiving antenna. On the other hand, the authors in [14-16] improved the spectral efficiency using SM and MIMO SCMA, which requires multiple antennas for the transmission and reception of the signals. In these methods the user bit stream is divided into two parts; one of them selects the antenna and the other part is mapped to codewords. Figure 7 shows the comparison of the method proposed in this work (SVD-SCMA) and the results shown in [16] for SM-SCMA and RGSM-SCMA using different numbers of antennas. 


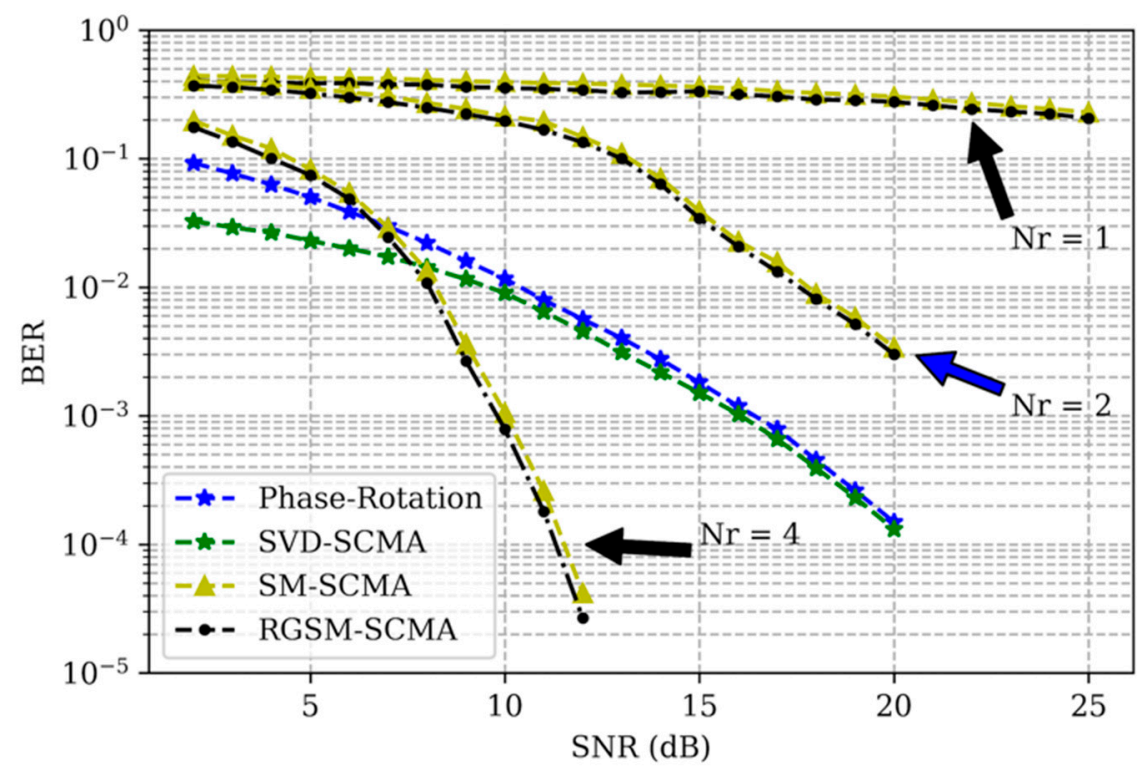

Figure 7. BER performance comparison.

As shown in this figure, SVD-SCMA performs better than SM-SCMA and RGSMSCMA with one and two receiving antennas. In the range from 0 to $+9 \mathrm{~dB}$, SVD-SCMA has a better performance than SM-SCMA and RGSM-SCMA, and for better channel conditions (above $8 \mathrm{~dB}$ SNR) the methods with four antennas have a better BER. It should be noted that SVD-SCMA uses a neural network-based receiver while SM-SCMA and RGSM-SCMA use MPA and ML for signal decoding.

\section{Conclusions}

In this paper we propose SVD-SCMA in conjunction with 8-APSK to improve the spectral efficiency of the uplink in 5G systems. The results show that SVD-SCMA has better BER with adverse SNR conditions $(<8 \mathrm{~dB})$ than algorithms using SM and MIMO for signal transmission. SVD-SCMA uses only one transmitting and receiving antenna, whereas previous works required antenna arrays with multiple radiating elements. The performance of the neural network receiver could be improved by modifying Adam $[24,28]$ with a long-term memory of the gradients used to update the parameters of the neural network adaptively.

The central hypothesis of this work is to increase the spectral efficiency (up to three bits) of the uplink through the design of codebooks (SVD-SCMA) and keeping the configuration of one transmitting and one receiving antenna. Preliminary tests that we have carried out show that with this configuration so far, it is not possible to increase the spectral efficiency to four bits, so the next step in this research is to use SVD-SCMA to encode three bits and use SM or MIMO to transmit more bits in the spatial plane and thus increase the number of bits transmitted simultaneously as well as using GPU to optimize receiver array operations. Combining SVD-SCMA in conjunction with SM/MIMO could increase spectral efficiency to more than 3 bits, resulting in a higher transmission rate for the eMBB segment of $5 \mathrm{G}$ communication systems and beyond.

Author Contributions: Conceptualization and methodology, S.V.-B.; mathematical modeling, J.L.L.B. and S.V.-B.; simulation and validation, S.V.-B. and J.L.L.-B.; writing-original draft preparation, S.V.-B.; editing, J.L.L.-B. All authors have read and agreed to the published version of the manuscript.

Funding: This research was funded by Instituto Politécnico Nacional, Mexico, Project number 20211324.

Institutional Review Board Statement: Not applicable for studies not involving humans or animals. Informed Consent Statement: Not applicable for studies not involving humans. 
Data Availability Statement: Data available on request. The data presented in this study are available on request from the corresponding author. The data are not publicly available because this work is part of an ongoing funded project.

Conflicts of Interest: The authors declare no conflict of interest.

\section{References}

1. ITU-R.IMT Vision-Framework and Overall Objectives of the Future Development of IMT for 2020 and Beyond, ITU-R M.2083-0. 2015. Available online: https:/ / www.itu.int/dms_pubrec/itu-r/rec/m/R-REC-M.2083-0-201509-I!!PDF-E.pdf (accessed on 16 March 2021).

2. Marsch, P.; da Silva, I.; Bulakci, O.; Tesanovic, M.; el Ayoubi, S.E.; Rosowski, T.; Kaloxylos, A.; Boldi, M. 5G radio access network architecture: Design guidelines and key considerations. IEEE Commun. Mag. 2016, 54, 24-32. [CrossRef]

3. Gupta, A.; Jha, R.K. A survey of 5G network: Architecture and emerging technologies. IEEE Access 2015, 1, 1206-1232. [CrossRef]

4. Dai, L.; Wang, B.; Yuan, Y.; Han, S.; Chih-Lin, I.; Wang, Z. Non-orthogonal multiple access for 5G: Solutions, challenges, opportunities, and future research trends. IEEE Commun. Mag. 2015, 53, 74-78. [CrossRef]

5. Bao, J.; Ma, Z.; Ding, Z.; Karagiannidis, G.K.; Zhu, Z. On the design of multiuser codebooks for uplink SCMA systems. IEEE Commun. Lett. 2016, 20, 1920-1923. [CrossRef]

6. Yu, L.; Lei, X.; Fan, P.; Chen, D. An optimized design of SCMA codebook based on star-QAM signaling constellations. In Proceedings of the International Conference on Wireless Communications Signal Processing (WCSP), Nanjing, China, 15-17 October 2015; pp. 1-5.

7. López-Bonilla, J.L.; Vidal-Beltrán, S.; Santiago-Rivera, I.; Martínez-Piñón, F. Construction of SCMA CodeBooks using the phase rotation method. In Proceedings of the IEEE International Autumn Meeting on Power, Electronics and Computing (ROPEC), Ixtapa, Mexico, 14-16 November 2018; pp. 1-8.

8. Liu, S.; Wang, J.; Bao, J.; Liu, C. Optimized SCMA Codebook Design by QAM Constellation Segmentation with Maximized MED. IEEE Access 2018, 6, 63232-63242. [CrossRef]

9. Yan, C.; Kang, G.; Zhang, N. A Dimension Distance-Based SCMA Codebook Design. IEEE Access 2017, 5, 5471-5479. [CrossRef]

10. Lou, S.; Gong, C.; Gao, Q.; Xu, Z. SCMA with Low Complexity Symmetric Codebook Design for Visible Light Communication. In Proceedings of the IEEE International Conference on Communications (ICC), Kansas City, MO, USA, 20-24 May 2018; pp. 1-6.

11. Vidal-Beltrán, S.; Carreno-Aguilera, R.; López Bonilla, J.L. Sparse Code Multiple Access Codebook Design Using Singular Value Decomposition. Fractals 2020, 28, 2150021. [CrossRef]

12. $\mathrm{Wu}$, X.; Haas, H.; Grant, P.M. Cooperative Spatial Modulation for Cellular Networks. IEEE Trans. Commun. 2018, 66, 3683-3693. [CrossRef]

13. Sinanović, D.; Šišul, G.; Kurdija, A.S.; Ilić, Ž. Multiple transmit antennas for low PAPR spatial modulation in SC-FDMA: Single vs. multiple streams. EURASIP J. Wirel. Commun. Netw. 2020, 2020, 44. [CrossRef]

14. Basar, E. Index modulation techniques for 5G wireless networks. IEEE Commun. Mag. 2016, 54, 168-175. [CrossRef]

15. Pan, Z.; Luo, J.; Lei, J.; Wen, L.; Tang, C. Uplink Spatial Modulation SCMA System. IEEE Commun. Lett. 2019, 23, 184-187. [CrossRef]

16. Al-Nahhal, I.; Dobre, O.A.; Basar, E.; Ikki, S. Low-Cost Uplink Sparse Code Multiple Access for Spatial Modulation. IEEE Trans. Veh. Technol. 2019, 68, 9313-9317. [CrossRef]

17. Mu, H.; Ma, Z.; Alhaji, M.; Fan, P.; Chen, D. A Fixed Low Complexity Message Pass Algorithm Detector for Up-Link SCMA System. IEEE Wirel. Commun. Lett. 2015, 4, 585-5888. [CrossRef]

18. Yang, L.; Liu, Y.; Siu, Y. Low complexity message passing algorithm for SCMA system. IEEE Commun. Lett. 2016, 20, 2466-2469. [CrossRef]

19. Bayesteh, A.; Nikopour, H.; Taherzadeh, M.; Baligh, H.; Ma, J. Low complexity techniques for SCMA detection. In Proceedings of the IEEE Globecom Workshops (GC Wkshps), San Diego, CA, USA, 6-10 December 2015; pp. 1-6.

20. Vidal-Beltrán, S.; López-Bonilla, J.L.; Martínez-Piñón, F. The Euclidean distance-based detection method applied on SCMA Codewords. Int. J. Latest Res. Sci. Technol. 2018, 7, 14-18.

21. Vidal-Beltrán, S.; Martínez-Piñón, F.; López-Bonilla, J.L. Supervised learning applied to the decoding of SCMA codewords. IEEE Latin Am. Trans. 2019, 17, 1843-1848. [CrossRef]

22. Huang, H.; Song, Y.; Yang, J.; Gui, G.; Adachi, F. Deep-Learning-Based Millimeter-Wave Massive MIMO for Hybrid Precoding. IEEE Trans. Veh. Technol. 2019, 68, 3027-3032. [CrossRef]

23. Baldi, M.; Chiaraluce, F.; de Angelis, A.; Marchesani, R.; Schillaci, S. A comparison between APSK and QAM in wireless tactical scenarios for land mobile systems. EURASIP J. Wirel. Commun. Netw. 2012, 2012, 317. [CrossRef]

24. Kingma, D.P.; Lei-Ba, J. Adam: A method for stochastic optimization. In Proceedings of the 2nd International Conference on Learning Representations, Banff, AB, Canada, 14-16 April 2014; Available online: https://arxiv.org/abs/1412.6980 (accessed on 17 March 2021).

25. Hu, Q.; Cai, Y.; Shi, Q.; Xu, K.; Yu, G.; Ding, Z. Iterative Algorithm Induced Deep-Unfolding Neural Networks: Precoding Design for Multiuser MIMO Systems. IEEE Trans. Wirel. Commun. 2021, 20, 1394-1410. [CrossRef] 
26. Yu, H. Network Complexity Analysis of Multilayer Feedforward Artificial Neural Networks. In Applications of Neural Networks in High Assurance Systems; Schumann, J., Liu, Y., Eds.; Studies in Computational Intelligence; Springer: Berlin/Heidelberg, Germany, 2010; Volume 268. [CrossRef]

27. Vidal-Beltrán, S.; López-Bonilla, J.L.; Martínez-Piñón, F.; Yalja-Montiel, J. Gradient Descent Optimization Algorithms for Decoding SCMA Signals. Int. J. Comput. Intell. Appl. 2020, 19, 2150002. [CrossRef]

28. Bock, S.; Wei, B.M. A Proof of Local Convergence for the Adam Optimizer. In Proceedings of the International Joint Conference on Neural Networks (IJCNN), Budapest, Hungary, 14-19 July 2019; pp. 1-8. [CrossRef] 